Elsevier

HRR 01208

\title{
Effect of electrical pulse shape on AVCN unit responses to cochlear stimulation
}

\author{
James A. Wiler, Ben M. Clopton, and Michael A. Mikhail \\ Kresge Hearing Research Institute, The University of Michigan, Ann Arbor, Michigan, U.S.A.
}

(Received 14 September 1988; accepted 20 December 1988)

\begin{abstract}
Electrical stimulation of the cochlea with a multiple-electrode array is best accomplished using pulsatile instead of continuous stimulation. The optimum shapes of electrical pulses for this purpose are still uncertain due to a lack of knowledge about their stimulation efficiency and requirements of the encoding strategy. We presented an extensive set of charge-balanced, rectangular pulse shapes to the guinea pig cochlea. Durations per phase for these constant-current pulses ranged from $20 \mu \mathrm{s}$ to $900 \mu \mathrm{s}$ with initially positive and initially negative polarities. Spike counts from single units in the anteroventral cochlear nucleus differed significantly for different pulse shapes, as did their initial latencies. Implications for stimulation efficiency and encoding strategies are discussed.
\end{abstract}

Cochlear nucleus; Electrical stimulation; Cochlear prosthesis; Single unit; Guinea pig

\section{Introduction}

Implanted cochlear prosthetic devices have been used for two decades to partially restore the sensation of hearing to profoundly deaf patients with intact VIIIth nerves. Waveforms used for electrical stimulation in clinical and experimental applications have ranged from relatively unprocessed versions of ambient sounds through sinusoids and pulses. The present strategy for improving prosthesis effectiveness is to restrict the spatial spread of excitation, creating as many independent "channels' as possible, and to maximize the rate of information transfer for each channel. Monopolar stimulation results in widespread excitation (van den Honert and Stypulkowski, 1987a); the most discrete stimulation is obtained with closely spaced bipolar electrodes placed on the surface of the bony spiral lamina (Merzenich et al., 1979; van den Honert and Stypulkowski, 1987a). If brief, biphasic pulses are delivered to arrays of bipolar electrodes properly situated in the cochlea, the

Correspondence to: James A. Wiler, Kresge Hearing Research Institute, 1301 E. Ann Street, Ann Arbor, MI 48109, U.S.A. potential exists for providing information at high rates. Pulses with a wide range of phase durations have been observed to be effective psychophysically (Shannon, 1981) and neurophysiologically (Hartmann et al., 1984; Parkins and Colombo, 1987; Parkins, 1987; Javel et al., 1987).

In addition to maximizing the rate of information transmission, minimizing the potential for damage is an important waveform criterion (Clopton et al., 1983). Due to electrochemical considerations (Brummer and Robblee, 1983), damage mechanisms are assumed to be proportional to charge density, the charge delivered across a fixed electrode surface. Neural and behavioral response measures for electrical stimulation have usually been expressed in terms of current (Pfingst, 1984; Clopton et al., 1983; Javel et al., 1987). When charge delivery for pulses and sinusoids is equated by taking the integral of current over their phase duration, i.e., the product of the RMS current and half period for sinusoids, it is apparent that thresholds are roughly proportional to the charge delivered per phase for short durations (Parkins and Colombo, 1987; Clopton and Bosma, 1982; Javel et al., 1987). Phase durations in excess of a few hundred $\mu$ s require increasing amounts of 
charge to reach threshold, at least for neural data (Parkins and Colombo, 1987; Clopton and Bosma, 1982). Behavioral thresholds indicate another range of minima for charge at threshold of 2-5 ms (Shannon, 1985), possibly attributable to central processing (Parkins and Colombo, 1987).

Our aim in this study was to examine the efficiency of different electrical pulse shapes delivered to the cochlea for evoking neural responses in the cochlear nucleus. The anteroventral cochlear nucleus (AVCN) was chosen as a recording site because its responses are comparable to axons of the VIIIth nerve, but electrical artifact was reduced and single neurons could be observed stably for longer periods as compared to the nerve. Since, at short pulse durations, neural activation appears to involve an integration of charge delivered (Simmons, 1966; Parkins and Colombo, 1987; Clopton and Bosma, 1982; Shannon, 1985), charge delivery during one phase of a pulse was kept constant, and only the shapes and initial polarities of pulses varied for a set of stimuli.

\section{Methods}

\section{Physiological preparation}

Thirteen pigmented guinea pigs weighing between 200 and $350 \mathrm{~g}$ were used for these observations. They were initially anesthetized with ketamine hydrochloride (Vetalar, $100 \mathrm{mg} / \mathrm{kg}$ ) and xylazine (Rompun, $5 \mathrm{mg} / \mathrm{kg}$ ) delivered intramuscularly and supplemented regularly to maintain appropriate levels of anesthesia. The skull was fixed with a high degree of stability to a rigid bar with a bolt embedded in dental acrylic surrounding stainless steel screws threaded into the dorsal cranium. In the initial experiments the external meatus was excised, and the bulla opened to allow an approach to the cochlea; the tympanic ring was broken, the tympanic membrane was removed, and the ossicles were disarticulated. Two holes approximately $0.2 \mathrm{~mm}$ apart were drilled in the second turn of the cochlea using a hand-rotated burr for placement of two teflon-coated, platinum-iridium ( $90 / 10 \%$ alloy), bipolar stimulating wires (Medwire, bare diameter of 0.005 inches). The stimulating wires were stabilized to the defect edge of the bulla with Durelon dental cement. Placements in later experiments were sometimes separated by greater distances, but stimulation was always bipolar with both electrodes within the cochlea. The electrode in phase with the stimulation waveform was designated the active one; the other electrode was the reference.

The posterior cranium was opened, and the cerebellum overlying the cochlear nucleus complex was aspirated to allow direct visual placement of the recording electrode on the dorsal surface. The entry point was on the rostral edge of the dorsal cochlear nucleus (DCN), angled rostrally towards the AVCN. To minimize the electrical artifact, a silver differential recording wire was placed on the dorsal surface of the brainstem near the recording electrode and stabilized to the cranial defect with Durelon. The animal was placed on a DC-powered heating pad, which maintained core body temperature at $37.5^{\circ} \mathrm{C}$ using feedback from a temperature probe. For several preparations, the hair cells were destroyed using a drop of concentrated neomycin sulfate solution infused into the round window while the scalar perilymph was aspirated. This causes a rapid disappearance of the cochlear microphonics in guinea pigs (Clopton and Bosma, 1982); thus, responses to electrical stimulation are the result of stimulation of neural elements and not those of hair cells (Clopton and Glass, 1984).

For some of the later preparations, the bulla was opened minimally, maintaining the integrity of the ossicles and tympanic membrane; this allowed for acoustical as well as electrical stimulation of the cochlea. The bipolar stimulating electrodes were placed in the cochlea at the level of the third turn, one in scala media and the other in scala vestibuli. The characteristic frequency (CF) of each unit was determined by presenting a $40 \mathrm{~ms}$ tone burst or a log-frequency sweep to the animal using a Beyer DT-48 earphone, enclosed in an aluminum coupler, and placed in direct contact with the external auditory meatus to form a closed system.

\section{Stimuli}

As shown in Fig. 1, the stimuli consisted of biphasic, rectangular pulses which were charge balanced; that is, the products of the phase duration and amplitude for both the positive and negative phases were equal. The parameters that were varied were the initial phase polarity (posi- 


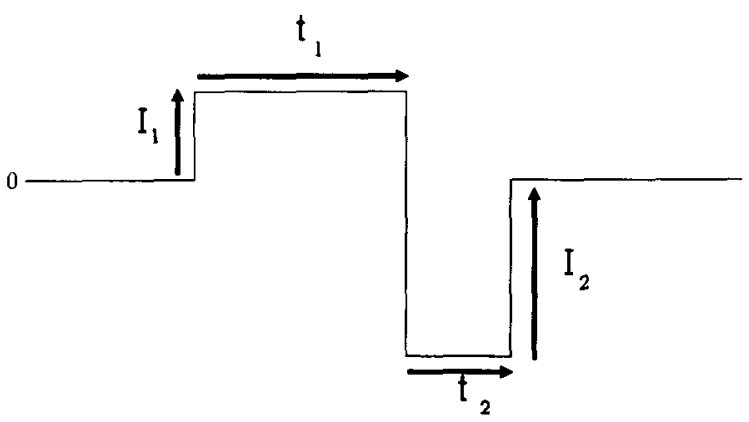

Fig. 1. Parameters for electrical pulses were specified in terms of currents $I_{i}$, durations $t_{i}$, and polarities for the first and second phases $(i=1,2)$. Specific pulse shapes are indicated by $\left[ \pm t_{1}, \mp t_{2}\right]$, phase durations in $\mu \mathrm{sec}$ and the polarities shown by the sign. The initial polarity (positive illustrated) was active relative to the reference electrode. Durations of the first and second phases satisfied charge balance $\left(I_{1} t_{1}+I_{2} t_{2}=0\right)$ within a pulse, constant absolute charge/phase transfer $\mathrm{C}$ over a set of pulses $\left(\mathrm{C}=\left|I_{1} t_{1}\right|=\left|I_{2} t_{2}\right|\right)$, a discrete time increment $\Delta t$ $\left(t_{i}=n_{i} \Delta t, n_{i}\right.$ an integer), and a maximum duration $T_{\max }$ $\left(t_{1}+t_{2} \leq T_{\max }\right)$. For a given set $k=1,2,3$, these parameters defined $N_{k}$ distinct pulse shapes, the product of two initial polarities and the number of allowable combinations of $t_{1}$ and $t_{2}$ as summarized in Table $I$.

tive or negative), the duration of each phase, and the overall magnitude of the pulses (the total absolute charge transfer). Specific pulse shapes will be referred to in terms of phase polarity and duration as explained in Fig. 1. The waveforms for the pulses were digitally synthesized with a $50 \mathrm{kHz}$ sample rate over a total of 8192 samples so that the pulses were contained within a total stimulus period of $163.84 \mathrm{~ms}$. This stimulus sequence was presented repeatedly over 200 cycles and a peristimulus-time histogram (PSTH) of the same duration was collected to summarize unit spike patterns, as discussed below.

Three different sets of pulses were synthesized to cover a wide range of pulse durations. Each set consisted of a group of stimulus files; in turn, each stimulus file consisted of eight different pulses in sequence over the period of $164 \mathrm{~ms}$ with $20 \mathrm{~ms}$ separation between pulses. The parameters defining the sets are shown in Fig. 1 and listed in Table I. The pulses in a stimulus file were drawn randomly from the entire set of $N_{k}$ possible pulse shapes $(k=1,2,3)$ so as to have different pulse durations, $T_{\max }$, presented in any given file, with the restriction that the initial phase of pulses in a given stimulus file was alternated between pulses and between each pulse file.

The first pulse set consisted of 90 different pulses ranging from 100 to $900 \mu$ s per phase, in $100 \mu \mathrm{s}$ intervals, with a maximum total pulse duration of $T_{\max }=1 \mathrm{~ms}$. These were arranged in 13 stimulus files with some redundancy to check on replication, specifically, the initial pulse in each file had a duration of $100 \mu$ s per phase alternating between a positive or negative first phase. The second pulse set consisted of 162 pulses and was an extension of the first. The durations of the phases were the same as before, but the maximum total duration of a pulse was unrestricted and thus ranged from $0.2-1.8 \mathrm{~ms}$. This added 72 pulses to the total number of pulses. The third pulse set consisted of 72 pulses ranging from 20 to $640 \mu \mathrm{s}$ per phase, in logarithmic intervals, as summarized in Table I. The initial pulse in each file had equal phase durations of $20 \mu \mathrm{s}$, and phase polarities alternated as described above.

All pulse stimuli were presented through an isolated constant-current stimulator built in-house. The stimulator had a voltage-to-current transformation ratio of $0.67 \mu \mathrm{A} / \mathrm{mV}$. Current was controlled by adjustment of the input voltage from the D-A converter to the stimulator with a manual attenuator. As shown in Fig. 1, the charge transfer during each phase of a pulse was constant for a stimulus presentation and given in nanocoulombs (nC) per phase. The peak current for either phase in $\mu \mathrm{A}$ is $I_{i}=10^{3} C_{p} / t_{i}$ where $C_{p}$ is the charge/phase in $\mathrm{nC}$ and $t_{i}$ is in $\mu \mathrm{s}$.

\section{Electrophysiological recording}

Glass micropipettes, $1 \mathrm{~mm}$ in diameter, were pulled, filled with $2 \mathrm{M}$ K-citrate, and beveled to give an impedance of 8-10 M $\Omega$ for AVCN single unit recordings. The brain tissue was covered with warm $2 \%$ agar-agar in physiologic saline to pre-

\section{TABLE I}

PULSE PARAMETERS DEFINING PULSE SETS

\begin{tabular}{lllll}
\hline Set & \multicolumn{1}{l}{$\Delta t$} & $T_{\max }$ & $n_{i}$ & \multicolumn{1}{l}{$N_{k}$} \\
\hline 1 & $100 \mu \mathrm{s}$ & $1000 \mu \mathrm{s}$ & $1,2,3, \ldots, 9$ & $(2 \times(9+9 \times 9)) / 2=90$ \\
2 & $100 \mu \mathrm{s}$ & $1800 \mu \mathrm{s}$ & $1,2,3, \ldots, 9$ & $2 \times 9 \times 9=162$ \\
3 & $20 \mu \mathrm{s}$ & $1280 \mu \mathrm{s}$ & $1,2,4,8,16,32$ & $2 \times 6 \times 6=72$ \\
\hline
\end{tabular}


vent dessication and reduce brain pulsations. The electrode was advanced from outside a doublewalled sound booth with a Trent Wells hydraulic microdrive until unit spikes were isolated which were larger than the stimulus artifact. Spike waveforms were discriminated in amplitude with a level comparator set to obtain maximum superimposition of triggered spike waveforms. The observed entry point on the dorsal surface, depth from micromanipulator readings and angle of penetration, were used to estimate electrode position within the nucleus.

Units were isolated using spontaneous activity if the cochlea was intact, but presentation of pulses was useful after the administration of neomycin, because spontaneous activity in the nucleus was reduced, although not necessarily eliminated, after this procedure. Following acquisition of a spike judged likely to exceed the electrical artifact during data collection, attenuation of the electrical pulses was reduced so that for the most effective pulses in the set, driving level was suprathreshold. Because minimization of the artifact was necessary, the stimulus level did not exceed the estimated threshold level by more than $5 \mathrm{~dB}$. Spike waveforms were monitored for artifact; if present, the trigger, stimulus level, or microdrive settings were adjusted in order to eliminate this con-

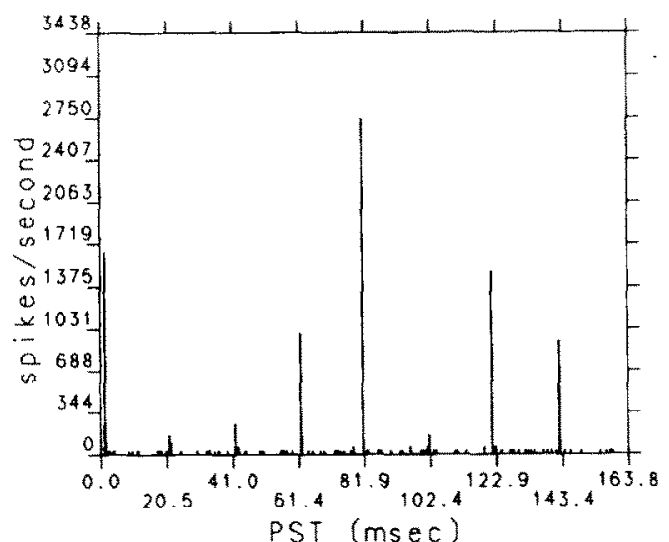

tamination, or the search for another unit was started.

\section{Data collection and analysis}

An AT-class 80286 processor with a Modular Instruments Incorporated laboratory interface was used for stimulus presentation and data collection. Each stimulus file was periodically presented as described above. PSTHs for 200 periods of each of the stimulus files were collected. Spike-event times were recorded with a clock accuracy of $10 \mu \mathrm{s}$ and summed in bins of $160 \mu \mathrm{s}$ for the displays and analyses in this study.

Each pulse set can be represented by a matrix of $(9+9 \times 9) / 2($ set 1$), 9 \times 9($ set 2$)$, or $6 \times 6$ (set 3) combinations of phase durations for both the positive and negative phases. Data are displayed in terms of these matrices, each representing data taken at one level of total charge.

Unit responses occurred stochastically with relatively short latencies following a pulse. An example of a PSTH to a sequence of 8 pulses comprising one stimulus file is shown in Fig. $2 \mathrm{~A}$. As illustrated in Fig. 2B, clusters of spike counts were seen after pulse onset with a minimum latency of about $1.4 \mathrm{~ms}$. Such measures of initial latency and total spike counts in the cluster make up the data reported here.

Fig. 2. A peristimulus-time histogram (PSTH) showing changes in spike rate evoked by different shapes. A) PSTH for a seguence of 8 different pulses, occurring at $20 \mathrm{~ms}$ intervals. The histogram is based on 200 periods of this stimulus file, a subgroup of stimulus set 1 . B) Enlargement of (A) to show the detail of the response to a single pulse from which spike count (total contributing to the major peak) and latency (time from pulse to initial bin of the response cluster) were estimated. 
The data are displayed as contour plots on the two-dimensional matrix for phase durations. The contours are parabolic interpolations of the data values obtained, as displayed by ISOX, a Fortran graphics program. The phase duration for the initial phase is on the ordinate, and that for the secondary phase on the abscissa. Spike counts are shown directly, but the reciprocal of latency (ms) is used so that larger values correspond to shorter latencies. Contour maxima can thus be viewed as indicating short-latency, high-probability spike occurrences.

\section{Results}

Fig. 2A shows an example of a PSTH collected in response to the first stimulus file of the first stimulus set and suggests the disparities in effectiveness for different pulse shapes. It was observed that the spike counts for unit responses in the AVCN differed greatly for different pulse shapes. While the effectiveness of each pulse shape also differed among units, similarities were seen across the stimulus sets for the most effective pulse shapes.

Spike-count and reciprocal-latency data contours were obtained for a total of 34 single units, where the recordings were judged to be free of artifact. Surface entry points, depth from surface, and, in some cases, acoustic driving agreed with unit sampling in the AVCN. We could not differentiate functional types within that division.

In general, maximal spike counts and minimal latencies were associated with durations of less
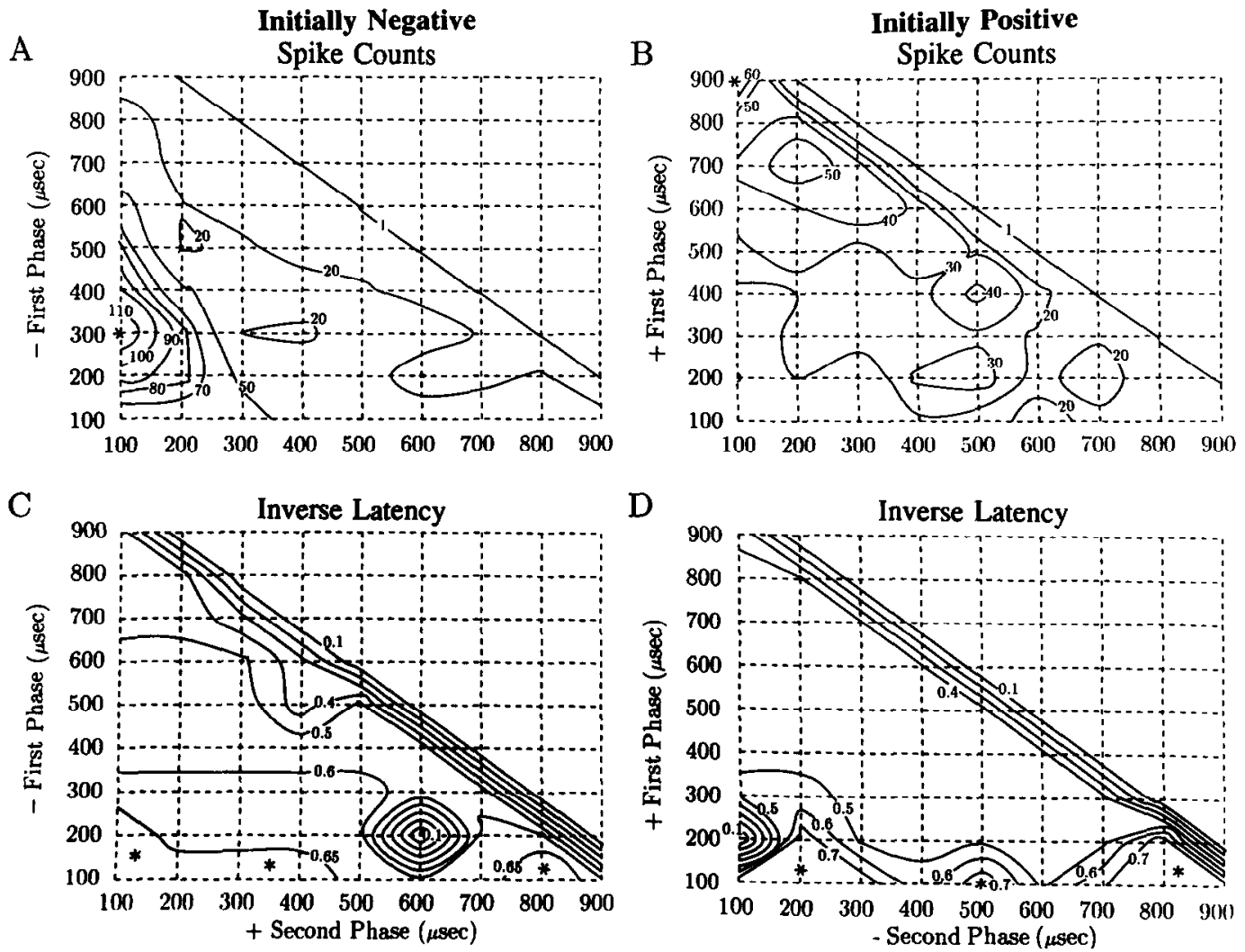

Fig. 3. Spike-count and reciprocal-latency contours for one unit in the AVCN driven by the first pulse set. Asterisks are used to mark maxima in this and following figures. Spike-count contours for a negative (A) first phase indicate selectivity for a cathodal phase of $300 \mu \mathrm{s}$ followed by a brief anodal phase of $100 \mu \mathrm{s}$. For a positive (B) first phase the optimal duration of the anodal phase is $900 \mu \mathrm{s}$ followed by a brief cathodal phase of $100 \mu \mathrm{s}$. Corresponding inverse-latency contours for the negative (C) and positive (D) first-phase conditions show that rapid spike occurrence tends to follow first phases of $100 \mu$ s duration independent of polarity. Electrodes in the second turn of the right cochlea, the reference electrode more apical. Charge transfer was $0.266 \mathrm{nC} / \mathrm{phase}$. 
than a few hundred $\mu \mathrm{s}$ for one or both phases. While most pulse shapes evoked some spikes over 200 presentations, the ratio of spikes evoked by the most effective shapes to those evoked by the least effective was often 50:1 or greater. For example, many pulses evoked only one spike over 200 presentations while a few usually evoked in excess of 50 , corresponding to a probability of spike generation of 0.05 and 0.25 , respectively. In some cases the most effective shapes evoked spikes with a probability in excess of 0.9 (180 spikes for 200 presentations) as opposed to an almost complete lack of responses to pulses with longer durations for both phases. Latencies for the onset of responses after a pulse were sometimes as little as $1.4 \mathrm{~ms}$, while other pulse shapes evoked responses with delays of $10 \mathrm{~ms}$ or more.
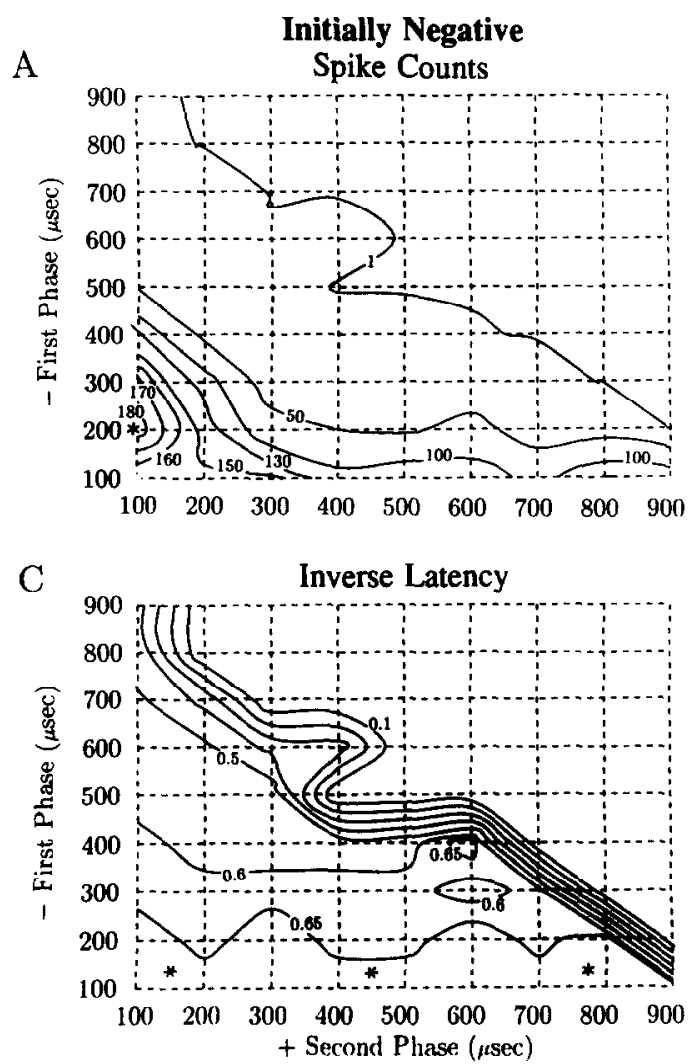

Another commonality was the relatively low charge transfer per phase needed to obtain driving. As shown in the following examples, pulsespecific driving was often obtained with less than $1.0 \mathrm{nC} /$ phase, although in some cases the required driving levels were higher (see Fig. 4). For the brief phase durations used in this study, the currents corresponding to these charge transfers were small, often less than $10 \mu \mathrm{A}$ peak.

For the first pulse set, shorter phase durations for one or both phases were most effective with few exceptions. Fig. 3 shows spike-count and reciprocal-latency contours for one unit in AVCN. As Fig. 3A illustrates, a shape of $[-300,+100]$ was much more effective in evoking spikes than other pulse shapes. When the first phase was positive (Fig. 3B), the $[+900,-100]$ shape was
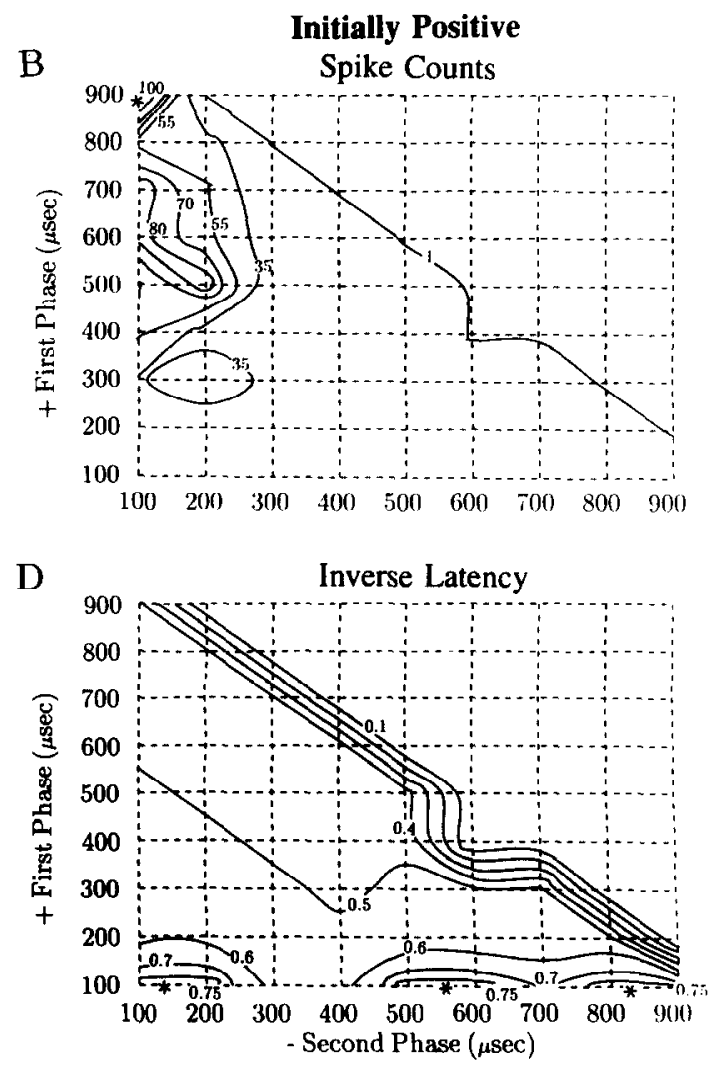

Fig. 4. Spike-count and reciprocal-latency contours for a unit in the AVCN of another preparation. As in Fig. 3, the first stimulus set was used. Conditions for the panels in this figure and those following correspond to those in the previous figure. These contours, although differing in detail from the previously described results, are very similar in their major features. The optimal pulse shape for an initially negative phase pulse was $[-200,+100](\mathrm{A})$ and the optimal pulse shape for an initially positive phase pulse was [ +900 , $-100]$ (B). Short initial phases are associated with the most rapid spike responses. Charge transfer was $59.6 \mathrm{nC} / \mathrm{phase}$. 
most effective in evoking spikes. In contrast, the inverse-latency contours of Figs. 3C and 3D show that a brief initial phase with duration of $100 \mu \mathrm{s}$ was associated with minimal latencies of 1.4-1.5 ms for many, although not all, values of positive phase duration. In short, the rapid delivery of charge associated with the initial phase condition evoked many spikes with least delay, regardless of the duration of the second phase. Notably, conditions for evoking the maximal spike count and minimal latency differed. A disassociation of shape parameters for maximal spike counts and minimal latencies was common over all units, but for both the spike and latency measures, phase durations falling below $100-200 \mu$ s for the initial phase were most effective. It should be noted that the 1.0-spike contour in panels $A$ and $B$ and the $0.1 / \mathrm{ms}$ contour in panels $\mathrm{C}$ and $\mathrm{D}$ for this and the next figure correspond to a lack of sample points above the negative diagonal of these matrices. Stimulus set 1 did not include these points.

Fig. 4 illustrates another variation in the response pattern observed to the first stimulus set. The charge transfer required to evoke a response was much larger than the previous figure, but again the initially negative pulse was most effective, the $[-200,+100]$ shape evoking spikes with a probability greater than 0.9 , as shown in panel A. For initially positive pulses, the $[+900,-100]$ shape evoked the most spikes (panel B), although it was less than for the best initially negative shape. In panels $C$ and $D$ the shortest initial phase
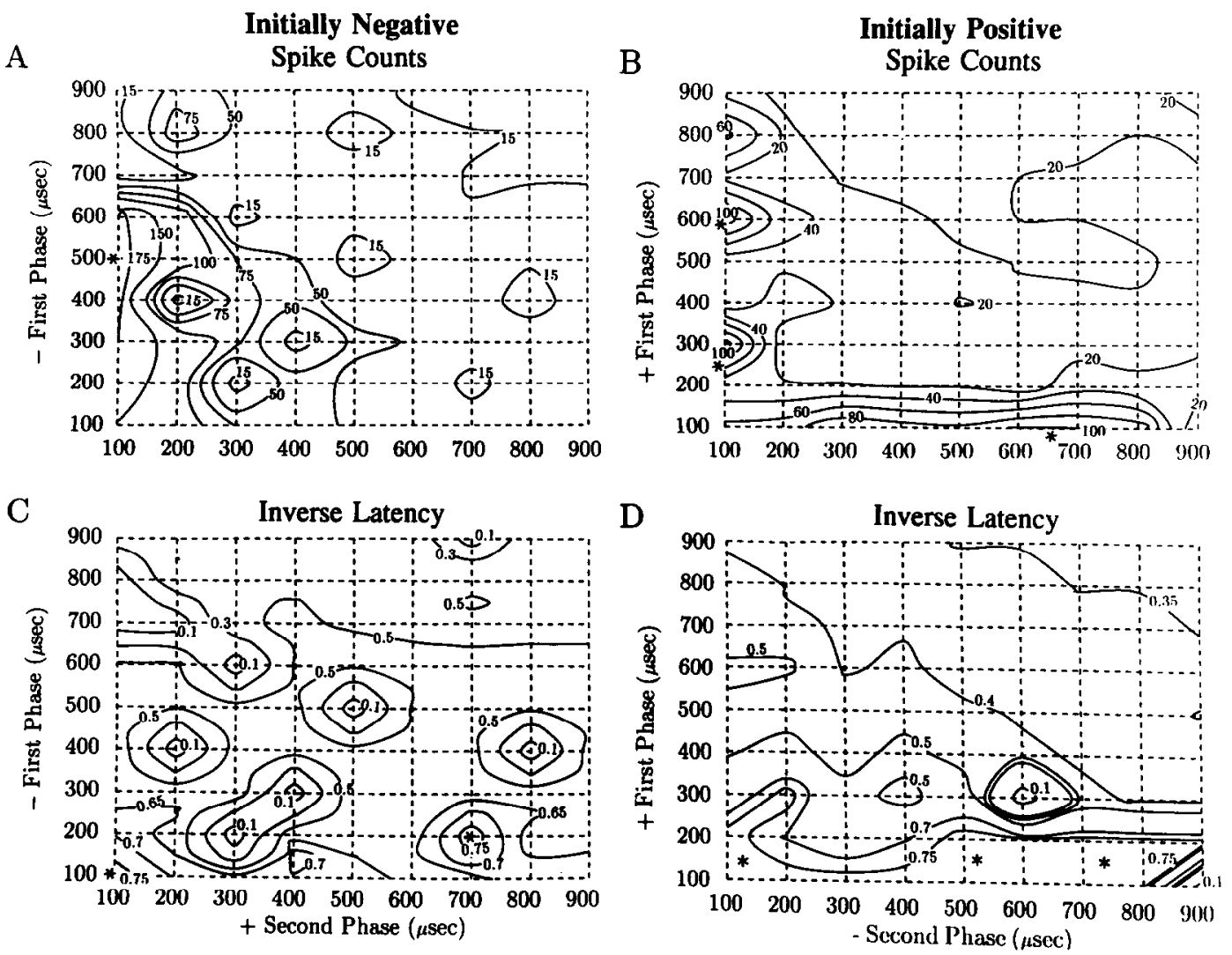

Fig. 5. Spike-count and reciprocal-latency contours for a unit in the AVCN driven by the second pulse set. For the initially negative phase condition (panels $A$ and $C$ ), the shapes of $[-400$ to $600,+100]$ were optimal for spike count, but $[-100,+100]$ and $[-200$, +700 ] evoked the most rapid spike occurrences after a pulse. The initially positive phase condition was less effective in generating spike counts (B), and durations of $100 \mu \mathrm{s}$ for both phases were optimal under some conditions. In panel, $\mathrm{D},-t_{2}=100 \mu \mathrm{s}$ was associated with short-latency responses. In general, the longer total pulse durations of set 2 did not indicate any novel effectiveness for the added shapes. Electrodes in second turn of left cochlea, reference electrode most basal. Charge transfer was $0.531 \mathrm{nC} / \mathrm{phase}$. 
is, as in Fig. 3, highly associated with minimal latencies.

Fig. 5 contains comparable data from another unit in a subsequent preparation, using the second pulse set (identical to the first pulse set, but without a limit on total duration). It should first be noted that contours for the second pulse set confirmed that pulses having total durations greater than 1.0 ms are relatively ineffective in evoking spikes; i.e., the restriction on set 1 did not eliminate effective pulse shapes. Some differences from the patterns of Figs. 3 and 4 are present. Panel $A$ with its optimal $[-500,+100]$ pulse is comparable to those in Figs. 3 and 4 , but a number of the initially positive pulses of panel $B$ are relatively effective, all having either a negative or positive phase duration of $100 \mu \mathrm{s}$ durations. Initially nega- tive pulses were most effective, producing larger spike counts with the maximum probability of firing being almost 0.9 as opposed to 0.5 for the best initially positive pulse. The inverse latencies of panels $C$ and $D$, as in the previous examples, indicate minimal latencies for brief initial phases of either polarity.

Examples of responses to the third set of stimuli are shown in Fig. 6. These logarithmically spaced matrices are in agreement with those from sets 1 and 2 but extend the observations to shorter phase durations. In panel $6 \mathrm{~A}$ the highest spike counts correspond to $[-160,+20]$ and $[-80,+160]$, whereas the initially positive phases of panel $6 \mathrm{~B}$ are most effective at $[+640,-20]$. These patterns are comparable to the previous ones. The latencies of panels $C$ and $D$ are also in agreement with
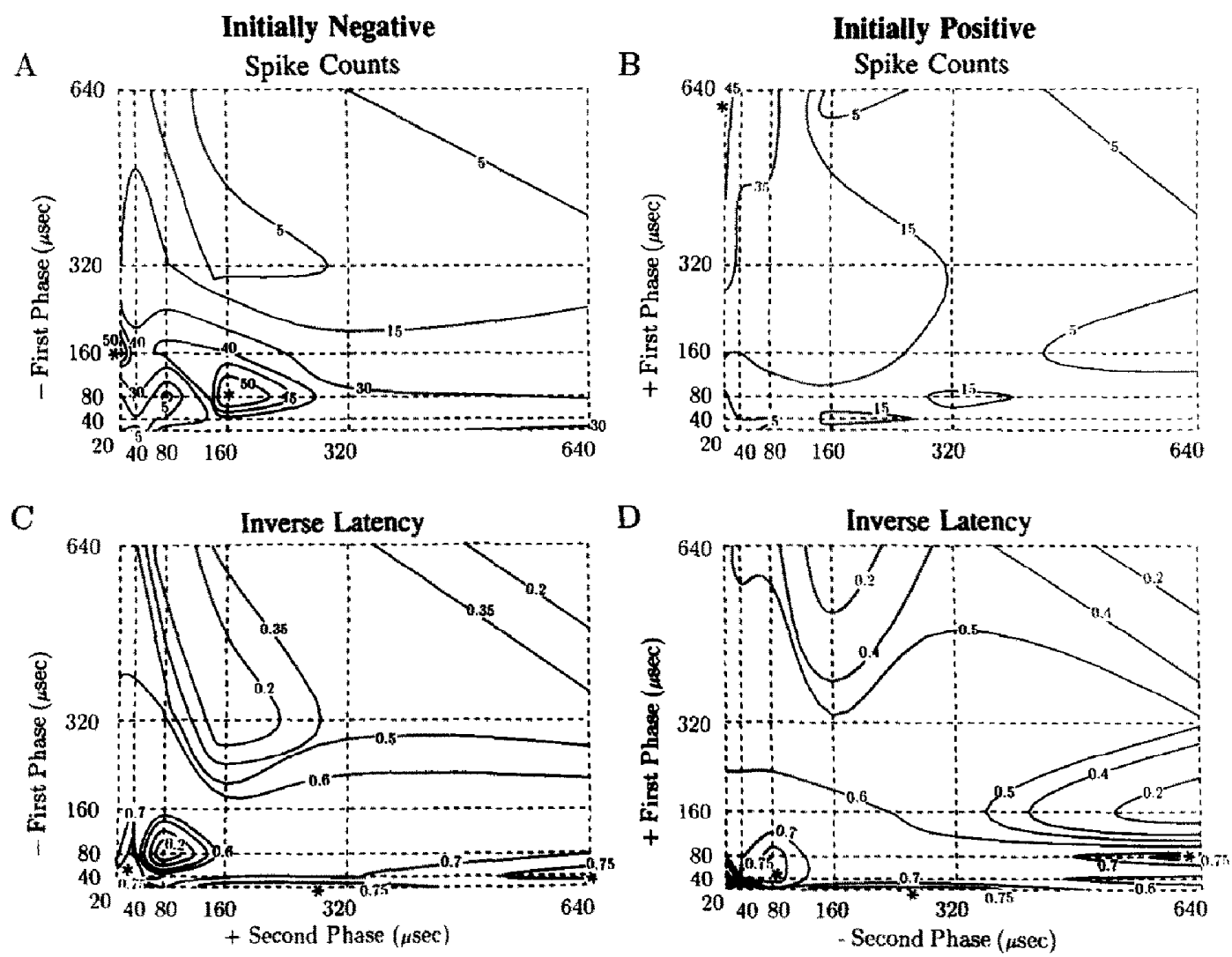

Fig. 6. Spike-count and reciprocal-latency contours for a unit in the AVCN driven by the third pulse set. Two shapes, [-80, +160$]$ and $[-160,+20]$, evoked high spike counts in the initially negative condition (A) while $[+640,-20]$ was most effective for the initially positive condition (B). Brief initial phases $(<160 \mu \mathrm{s})$ were associated with rapid spike occurrences under both polarity conditions ( $C$ and $D$ ). Electrode in left cochlea, active on the round window, reference in third turn. Cochlea was acoustically stimulated; unit CF was $655 \mathrm{~Hz}$ and had a primary-like, phase-locked PSTH to tone bursts. Charge transfer was $0.267 \mathrm{nC} /$ phase. 
previous figures in that they are minimal for initial-phase durations of less than $160 \mu \mathrm{s}$.

These examples were representative of all of the units observed and illustrate some of the commonly observed variations. Preparations with intact tympanic membranes had similar responses to the electrical stimulation as the previous preparations. The characteristic frequencies of the units ranged from 650 to $740 \mathrm{~Hz}$ and PSTHs tended to be primary-like in shape. Electrode placements for these units tended to be more apical, so lower characteristic frequencies for electrically activated units would be expected. No differences in response profiles were seen between the preparations in which the hair cells were destroyed with neomycin sulfate and those with intact hair cells.

\section{Discussion}

These observations bear on two considerations critical to the operation of a cochlear prosthesis; conveyance of information to the auditory system by electrical stimulation and minimization of tissue damage. The results indicate that there is an optimal shape of charge-balanced pulses for driving units in the cochlear nucleus that depends on the polarity of the initial phase. It is accepted that electrical activation of the cochlea is achieved by depolarization of neural membranes (Clopton and Bosma, 1982; Colombo and Parkins, 1987; van den Honert and Stypulkowski, 1987a; van den Honert and Stypulkowski, 1987b; Javel et al., 1987). It is also apparent that both cathodal and anodal pulses can evoke responses but that cathodal pulses depolarize membranes locally and thereby more efficiently. Electrode orientation is also important (van den Honert and Stypulkowski, 1987a). Given previous work, it is apparent that polarity should be referenced to the electrode nearest the site of stimulation, except for roughly equidistant bipolar pairs where polarity effects become highly dependent on the electrical field and neuronal geometries (Ranck, 1975). In studies, such as this, where identification of the effective cathode is not possible due to closely spaced electrodes and an unknown site of membrane activation, we assume that a cathodal phase preceding an anodal phase is the more effective stimulus (van den Honert and Stypulkowski, 1987a,b). From this study, an initially cathodal phase of 200-300 $\mu$ s followed by an anodal phase of $100 \mu \mathrm{s}$ or less was the more effective pulse shape. If the anodal phase preceded the cathodal, a very long anodal phase, $800-900 \mu \mathrm{s}$, was most effective provided that the duration of the second phase was brief, $100 \mu \mathrm{s}$ or less.

Variations on this pattern (e.g., Figs. 5 and 6) did occur and were probably influenced by two important factors; electrode placement relative to the site of afferent stimulation and the determinants of unit response in AVCN. Some variants of the response patterns were those to be expected from an interchange of the effective initial-phase cathode and anode, a predictable result from a sampling of units in the AVCN which are driven by varied spatial sites in the cochlea relative to the electrodes. Other variations are more difficult to interpret and may have arisen because of the nature of afferent connectivity on neurons of the AVCN. Panel B of Fig. 5 and panel A of Fig. 6 show examples of more than one pulse shape evoking more spikes than the immediately surrounding shapes. Multiple afferents with different relationships to the stimulating electrodes or disparate selectivities for pulse parameters might underlie these patterns. Records from primary afferents could resolve this question, although problems with electrical artifact and duration of the stimulus profile would complicate data collection (Parkins and Colombo, 1987).

Asymmetries in the rate of charge transfer between pulse phases have not been investigated in the auditory system, but previous work on electrical stimulation of neural elements suggests that this is a parameter influencing stimulation efficiency (Lilly, 1961; Ranck, 1975; Loeb et al., 1983). In this study, pulses with specific asymmetries were observed to clearly evoke more unit spikes for a given charge transfer than any other waveform shape in the class of rectangular pulses. Current density, $\mu \mathrm{A} / \mathrm{cm}^{2}$, is greater for shorter pulse durations, but damage mechanisms have been related to charge density, $\mu \mathrm{C} / \mathrm{cm}^{2}$, based on electrochemical considerations. Specifically, Faradic reactions have been shown to occur after the breakdown of a double-layer capacitance at the electrode-electrolyte interface when the charge density exceeds more than a few $\mu \mathrm{C} / \mathrm{cm}^{2}$ 
(Brummer and Robblee, 1983). Estimates of damaging densities for $\mathrm{Pt}$ electrodes run as high as $300-400 \mu \mathrm{C} / \mathrm{cm}^{2}$, but biological observations (Duckert and Miller, 1982; Leake-Jones et al., 1980) suggest the possibility of damage in the $10-100 \mu \mathrm{C} / \mathrm{cm}^{2}$ range for phase durations of 200-500 $\mu \mathrm{s}$.

Charge transfer per phase at threshold for measures of neural response has varied but tends to fall in the range of $4-150 \mathrm{nC} /$ phase for brief, biphasic pulses and high-frequency sinusoids (Clopton and Bosma, 1982; Clopton et al., 1983; Hartmann et al., 1984; Parkins and Colombo, 1987; Javel et al., 1987). Radial placements for bipolar electrodes by van den Honert and Stypulkowski (1987a) indicate, in some cases, charge transfers at threshold of significantly less than $1 \mathrm{nC} /$ phase for $100 \mu \mathrm{s}$ cathodal monophasic pulses. Behavioral measures tend to be associated with somewhat lower values than most neural measures (Simmons, 1966; Shannon, 1985), some falling below the $1 \mathrm{nC}$ /phase level (Pfingst et al., 1979). The optimal pulse shapes suggested by this study were effective at charge densites of less than $0.5 \mathrm{nC} /$ phase. At $0.5 \mathrm{nC} /$ phase, a half-spherical intracochlear electrode of $100 \mu \mathrm{m}$ diameter, such as is commonly obtained by flaming $\mathrm{Pt}-\mathrm{Ir}$ wire, would have a charge density per phase of 1.59 $\mu \mathrm{C} / \mathrm{cm}^{2}$ based on geometric surface area. Since the real surface area would be $30-40 \%$ greater (Brummer and Robblee, 1983), the actual charge density would be even less. Optimization of pulse shape may provide significant protection against tissue damage due to high charge densities.

Utilization of pulse shape to vary stimulation efficiency offers some flexibility in how information delivery is approached. While parameter control is usually directed at obtaining the highest probability of neural spike generation, it could also provide a more stochastic spike generation. The second strategy would use pulse shape to span a range of pulse efficiencies. While it is intuitive to choose the most effective shape on the basis of the greatest spike count evoked, increasing the stochasticity of electrical driving, that is, evoking spikes with a lower probability per pulse, might have some advantage, since this more closely resembles the normal representation of acoustic signals (Dobie and Dillier, 1985).
Pulse parameters affect encoding strategies by limiting the rate of stimulation and possibilities for pulse interaction both in time and across an array of electrodes. The general goal is a sequence of phase durations and intensities that, while maintaining charge balance, result in spatially separate and functionally independent spike generation in VIIIth nerve fibers. For low rates of pulse delivery, an initially cathodal phase with a duration of $200-300 \mu \mathrm{s}$ followed by a brief anodal phase of $100 \mu \mathrm{s}$ or less is the most efficient pulse shape on average. For higher rates of pulse generation on a single electrode, anodal phases must immediately precede cathodal phases if the interpulse interval is less than $1 \mathrm{~ms}$. The results of this study suggest that the effective cathodal phase should be kept brief, $100 \mu \mathrm{s}$ or less, and the anodal phase lengthened to $500 \mu \mathrm{s}$, or more, if possible.

\section{Acknowledgements}

This work was supported by grant NS21440 from the National Institutes of Health. We would like to thank Dr. K.-P. Beier for providing the contour program ISOX for the Apollo computers.

\section{References}

Brummer, S.B. and Robblee, L.S. (1983) Criteria for selecting electrodes for electrical stimulation: Theoretical and practical considerations. Ann. N.Y. Acad. Sci. 40, 159-171.

Clopton, B.M. and Bosma, M.A. (1982) Effectiveness of middle ear electrical stimulation for activating central auditory pathways. Ann. Otol. Rhinol. Laryngol. 91, 285-291.

Clopton, B.M., Spelman, F.A., Glass, I., Pfingst, B.E., Miller, J.M., Lawrence, P.D. and Dean, D.P. (1983) Neural encoding of electrical signals. Ann. N.Y. Acad. Sci. 40, 146-158.

Clopton, B.M. and Glass, 1. (1984) Unit responses at cochlear nucleus to electrical stimulation through a cochlear prosthesis. Hear. Res. 14, 1-14.

Dobie, R.A. and Dillier, N. (1985) Some aspects of temporal coding for single-channel electrical stimulation of the cochlea. Hear. Res. 18, 41-55.

Duckert, L.G. and Miller, J.M. (1982) Acute morphological changes in guinea pig cochlea following electrical stimulation. Ann. Otol. Rhinol. Laryngol. 91, 33-40.

Hartmann, R, Topp. G. and Klinke, R, (1984) Discharge patterns of cat primary auditory fibers with electrical stimulation of the cochlea. Hear. Res. 13, 47-62.

van den Honert, C. and Stypulkowski, P.H. (1987a) Single fiber mapping of spatial excitation patterns in the electrically stimulated auditory nerve. Hear. Res. $29,195-206$. 
van den Honert, C. and Stypulkowski, P.H. (1987b) Temporal response patterns of single auditory nerve fibers elicited by periodic electrical stimuli. Hear. Res. 29, 207-222.

Javel, E., Tong, Y.C., Shepherd, R.K. and Clark, G.M. (1987) Responses of cat auditory nerve fibers to biphasic electrical current pulses. Ann. Otol. Rhinol. Laryngol. (St. Louis). Suppl. 128. 13, 26-30.

Leake-Jones, P.A., O'Reilly, B.F. and Vivion, M.C. (1980) Neomycin ototoxicity: Ultrastructural surface pathology in the organ of Corti. SEM/1980/III: 427-434. SEM, Inc., AMF O'Hare, IL 60660.

Lilly, J.C. (1961) Injury and excitation by electric currents. A. The balanced waveform. In: D.W. Sheer, (Ed.), Electrical Stimulation of the Brain, Texas University Press, Austin, Texas, pp. 60-64.

Loeb, G.E., White, M.W, and Jenkins, W.M. (1983) Biophysical considerations in electrical stimulation of the auditory nervous system. Ann. NY. Acad. Sci. 405, 123-136.

Merzenich, M.M., White, M., Vivion, M.C., Leake-Jones, P.A. and Walsh. S. (1979) Some considerations of multichannel electrical stimulation of the auditory nerve in the profoundly deaf; interfacing electrode arrays with the auditory nerve array. Acta. Otolaryngol. 87, 196-203.
Parkins, C.W. and Colombo, J. (1987) Auditory-nerve singleneuron thresholds to electrical stimulation from scala tympani electrodes. Hear. Res. 31, 267-286.

Parkins, C.W. (1987) Single auditory neuron response patterns to different implant stimulus wave forms. Ann. Otol. Rhinol. Laryngol. Suppl 128. 96, 41-42.

Pfingst, B.E., Miller, J.M., Donaldson, J.A. and Spelman, F.A. (1979) Psychophysical evaluation of cochlear prostheses in a monkey model. Ann. Otol. Rhinol. Laryngol. 88, 613-625.

Pfingst, B.E. (1984) Operating range and intensity psychophysics for cochlear implants. Arch. Otolaryngol. 110, 140-144.

Ranck, J.B., Jr. (1975) Which elements are excited in electrical stimulation of mammalian central nervous system: A review. Brain Res. 98, 417-440.

Shannon, R.V. (1981) Growth of loudness for sinusoidal and pulsatile electrical stimulation. Ann. Otol. Rhinol. Laryngol. Suppl 82. 90, 13-14.

Shannon, R.V. (1985) Threshold and loudness functions for pulsatile stimulation of cochlear implants. Hear. Res. 18, 135-143.

Simmons, F.B. (1966) Electrical stimulation of the auditory nerve in man. Arch. Otolaryngol. 84, 24-76. 